\title{
Nurses' delivery of the Tobacco Tactics intervention at a Veterans Affairs Medical Center
}

\author{
Amanda M Fore, Carrie A Karvonen-Gutierrez, AkkeNeel Talsma and Sonia A Duffy
}

\begin{abstract}
Aims and objectives. To determine (1) factors associated with nurses' perceived confidence in and importance of delivering cessation interventions to patients after receiving the Tobacco Tactics educational module, and (2) whether self-reported delivery of smoking cessation services increased after the Tobacco Tactics educational programme was implemented.

Background. Intensive nurse-based inpatient smoking cessation interventions are effective; however, due to a lack of nurse confidence, training and time, nurseadministered cessation interventions are seldom implemented.

Design. Two cross-sectional surveys among staff trained in the Tobacco Tactics programme, conducted at two months and 15 months post-training.

Methods. Surveys were conducted to determine whether self-reported delivery of smoking cessation services by nursing staff increased after delivery of the Tobacco Tactics training at a Midwestern Veterans Affairs Medical Center. All staff members who attended the training were eligible to complete the surveys at two and 15 months post-training.

Results. Having a good understanding of the elements of smoking cessation interventions and satisfaction with training were associated with perceived confidence and importance of delivering smoking cessation interventions. Additionally, $86 \%$ of participants reported delivering cessation interventions 15 months post-training compared with $57 \%$ prior to training ( $p<0.0001)$.

Conclusions. Training nurses how to deliver tobacco cessation interventions increases delivery of cessation services.

Relevance to clinical practice. Nurse-delivered cessation interventions have the potential to increase quit rates and decrease morbidity and mortality among patient populations.
\end{abstract}

Key words: implementation, nurse education, smoking

Accepted for publication: 16 July 2013

Authors: Amanda M Fore, MS, RN, Nurse Coordinator/Program Analyst, National Center for Patient Safety, MI; Carrie A Karvonen-Gutierrez, MPH, Research Health Science Specialist, Department of Health Services Research and Development, Ann Arbor VA Center for Clinical Management Research, MI; AkkeNeel Talsma, PhD, RN, FAAN, Research Investigator, School of Medicine, University of Michigan, MI; Sonia A Duffy, PhD, RN, FAAN, Research Scientist and Professor, Department of Health Services Research and Development, Ann Arbor VA Center for Clinical Management Research and Departments of Otolaryngol-
What does this paper contribute to the wider global clinical community?

- Having a good understanding of the elements of smoking cessation interventions and satisfaction with training are associated with perceived confidence and importance of delivering smoking cession interventions.

- Training nurses how to deliver tobacco cessation interventions increases delivery of cessation services.

- Nurse-delivered cessation interventions have the potential to increase quit rates and decrease morbidity and mortality among patient populations. ogy and Psychiatry, School of Nursing, University of Michigan, Ann Arbor, MI, USA

Correspondence: Sonia A Duffy, Research Scientist and Professor, Department of Health Services Research and Development, Ann Arbor VA Center for Clinical Management Research, Ann Arbor, MI, USA and Departments of Otolaryngology and Psychiatry, School of Nursing, University of Michigan, 2215 Fuller Rd. Ann Arbor, MI 48105, USA. Telephone: +1 7343950613.

E-mail: bump@umich.edu 


\section{Introduction}

Smoking rates among veterans are higher than the general population (Office of Quality \& Performance 2001, Centers for Disease Control \& Prevention 2007). In the Department of Veterans Affairs (VA) Veterans Integrated Service Network (VISN), 11, 28.2\% of veterans are current smokers compared with $20.9 \%$ of the general population (Department of Veterans Affairs 2006). Duffy et al. (2008) suggest that $70 \%$ of veteran smokers reported a desire to quit; however, only $17 \%$ received smoking cessation services while hospitalised at a VISN 11 Medical Centre. Consequently, an implementation study, known as Tobacco Tactics, was initiated to disseminate a nurse-delivered smoking cessation programme to Veteran inpatients in two VISN 11 VA Medical Centres (Duffy et al. 2010). Although this study was conducted at two hospitals in one geographical area in the USA, smoking remains a global problem, and nurses throughout the international community are in a unique position to implement smoking cessation strategies.

\section{Background}

A 2005 Cochrane Collaborative Review on smoking cessation services delivered to patients being treated on inpatient units showed that highly intensive interventions with at least one month of follow-up were effective (Rigotti et al. 2002). Additionally, in 2011, the Joint Commission released standards that apply to all hospitalised smokers and include the following: (1) tobacco use screening, (2) treatment provided or offered during the hospital stay, (3) treatment provided or offered at discharge and (4) assessment of tobacco use status within 30 days after hospital discharge (Joint Commission 2011). Hospitalisation is an opportune time to deliver cessation services because patients are a captive audience, they are removed from daily cues to smoke, are often motivated to quit due to illness and have temporarily quit due to smoking bans (Smith et al. 1999).

A Cochrane Collaborative meta-analysis has shown that smoking cessation advice and/or counselling given by nurses to patients is efficacious (Rice \& Stead 2008). Nurses are ideally positioned to provide cessation interventions because they: (1) are educated in patient education, psychosocial interventions and physiological interventions, (2) have access to and immediate rapport with patients and (3) understand patients' medical condition and can tailor the intervention accordingly. The remaining challenge is incorporating effective smoking cessation interventions into standard practice, as a large gap exists between the availability of these interventions and their widespread dissemination and implementation (Taylor \& Curry 2004).

Although nurses generally have a positive attitude concerning cessation services and are in an ideal position to provide such services, many frequently do not deliver cessation interventions (McCarty et al. 2001, Casey 2007). While patient motivation and lack of time are cited as high-frequency barriers limiting the delivery of cessation interventions in clinical practice, lack of skills and knowledge on how to help patients quit closely follows (Sarna et al. 2001). Cross-sectional surveys of nurses have shown that self-efficacy (confidence) and perceived importance of counselling are cited as barriers to delivering tobacco cessation counselling (Borrelli et al. 2001, Sarna et al. 2001) and are related to lack of training in cessation interventions, which is rarely offered in nursing curricula (Wewers et al. 2004). Despite the fact that the importance of educating providers in cessation interventions has been noted in the literature (Campbell et al. 2011), once nurses are trained in cessation interventions, little is known about nurses' perceptions and delivery of cessation services (Taylor \& Curry 2004). Hence, the objectives of this study were to determine: (1) factors associated with nurses' perceived confidence in and importance of delivering cessation interventions to patients after receiving the Tobacco Tactics educational module and (2) whether self-reported delivery of smoking cessation services increased after the Tobacco Tactics educational programme was implemented.

\section{Methods}

\section{Design}

The implementation of the Tobacco Tactics study in the VA is described in a prior published study (Duffy et al. 2010). As part of this larger study, two cross-sectional surveys of nurses and other staff were conducted two months after participating in the Tobacco Tactics training and again 15 months post-training. Human subjects approval was received from the VA in which the research was conducted.

\section{Sample}

The Tobacco Tactics training was provided to 333 clinical staff, of which $85 \%$ were registered nurses (RNs) or licensed practical nurses (LPNs), who provide care to patients at a Midwestern VA Medical Center. Other participants included nursing students, nursing assistants, physicians, respiratory therapists and social workers. The one-hour mandatory training for nurses was offered during a four-month period in 2007 and during monthly orientation sessions for new 
nurses. Nurses received one continuing education credit (CEU) for participating in the training. All staff members who attended the training were eligible to complete a survey two months post-training (to measure proximal response to training) and then again 15 months post-training (to measure distal response to the training). Anonymous staff surveys, along with return envelopes, were delivered to employee mailboxes or through interdepartmental mail. Because the response population differed for the two surveys and unique identifiers were not provided on the surveys, individual differences between the two time points could not be examined. The response rate was 49\% (145/298) for the two-month survey and 90 staff returned the 15 -month survey. The response rate for the 15 -month survey is unknown as the total number of surveys provided was not recorded.

Demographic and smoking history information of the staff who completed the two- and 15-month post-training surveys is included in Table 1 . Less than $10 \%$ of the samples were current smokers, nearly two-thirds were $>45$ years old, and over two-thirds had a four-year college degree. Most were female, White and worked on an acute care unit. Additional demographics are displayed in Table 1.

\section{Intervention}

The Tobacco Tactics toolkit for nurses contains the following: (1) one contact hour for training, (2) a one-hour PowerPoint presentation on behavioural and pharmaceutical interventions, (3) a pocket card 'Helping Smokers Quit: A Guide for Clinicians' developed by the US Department of Health and Human Services Public Health Service and Tobacco Free Nurses, (4) evidence-based behavioural and pharmaceutical protocols (Table 2) (Duffy et al. 2010) and (5) computerised template for nurse documentation.

The evidence-based intervention uses the Tobacco Tactics toolkit for patients, which includes a: (1) brochure, (2) educational video on smoking cessation, (3) Tobacco Tactics manual, (4) pharmaceuticals, (5) 1-800-QUIT-NOW helpline and (6) follow-up phone calls. A cessation brochure is provided to all smokers. For those interested in cessation, the educational programme, 'Smoking: Getting Ready to Quit', can be viewed on the hospital's education channel or by videotape. The Tobacco Tactics manual is written at an eighthgrade reading level, with large print, colour pictures, stories and written exercises for patients. Providing the brochure, videotape and manual in advance saves the nurses' time; nurses spend 10-20 minutes counselling patients using the pieces of the toolkit. The pharmaceutical protocol (Table 2) included an algorithm for providing: (1) nicotine replacement therapy (NRT), (2) bupropion, (3) combination of NRT and
Table 1 Nurses' smoking history and demographic variables

\begin{tabular}{|c|c|c|}
\hline & $\begin{array}{l}\text { Two-month } \\
\text { post-training } \\
\text { assessment } \\
n(\%)\end{array}$ & $\begin{array}{l}\text { Fifteen-month } \\
\text { post-training } \\
\text { assessment } \\
n(\%)\end{array}$ \\
\hline \multicolumn{3}{|l|}{ Smoking status } \\
\hline Current & $11(7 \cdot 8)$ & $4(4 \cdot 8)$ \\
\hline Former & $52(36 \cdot 6)$ & $31(36 \cdot 9)$ \\
\hline Never & $79(55.6)$ & $49(58.3)$ \\
\hline \multicolumn{3}{|l|}{ Age (years) } \\
\hline$<35$ & $23(16 \cdot 3)$ & $14(19 \cdot 2)$ \\
\hline $35-44$ & $29(20 \cdot 6)$ & $12(16 \cdot 4)$ \\
\hline $45-54$ & $57(40 \cdot 4)$ & $27(37 \cdot 0)$ \\
\hline $55-64$ & $30(21 \cdot 3)$ & $19(26 \cdot 0)$ \\
\hline$>64$ & $2(1.4)$ & $1(1.4)$ \\
\hline \multicolumn{3}{|l|}{ Sex } \\
\hline Male & $20(14 \cdot 3)$ & $13(16 \cdot 3)$ \\
\hline Female & $120(85 \cdot 7)$ & $67(83 \cdot 8)$ \\
\hline \multicolumn{3}{|l|}{ Race } \\
\hline White & $100(80 \cdot 7)$ & $66(84.6)$ \\
\hline Black & $16(12 \cdot 9)$ & $5(6.4)$ \\
\hline Other & $8(6 \cdot 5)$ & $7(9.0)$ \\
\hline $\begin{array}{l}\text { Have four-year college } \\
\text { degree }\end{array}$ & $89(66.9)$ & $56(70 \cdot 9)$ \\
\hline \multicolumn{3}{|l|}{ Unit } \\
\hline Inpatient psychiatry & $13(10 \cdot 7)$ & $10(13 \cdot 3)$ \\
\hline Outpatient psychiatry & $7(5 \cdot 7)$ & $5(6 \cdot 7)$ \\
\hline Surgery & $11(9 \cdot 0)$ & $3(4 \cdot 0)$ \\
\hline $\begin{array}{l}\text { Inpatient oncology, } \\
\text { medicine, telemetry, } \\
\text { cardiac }\end{array}$ & $32(26 \cdot 2)$ & $21(28 \cdot 0)$ \\
\hline Med ECC/nursing home & $12(9 \cdot 8)$ & $6(8 \cdot 0)$ \\
\hline $\begin{array}{l}\text { Intensive care units } \\
\text { (MICU, TICU, SICU) }\end{array}$ & $19(15 \cdot 6)$ & $15(20 \cdot 0)$ \\
\hline Ambulatory care & $7(5 \cdot 7)$ & $7(9 \cdot 3)$ \\
\hline $\begin{array}{l}\text { Outpatient (respiratory, } \\
\text { endoscopy, radiology, } \\
\text { IV team) }\end{array}$ & $13(10 \cdot 7)$ & $4(5 \cdot 3)$ \\
\hline $\begin{array}{l}\text { Nonpatient care (research, } \\
\text { quality management, } \\
\text { medical care cost recovery) }\end{array}$ & $4(3 \cdot 3)$ & $1(1 \cdot 3)$ \\
\hline Inpatient house supervisors & $4(3 \cdot 3)$ & $3(4 \cdot 0)$ \\
\hline
\end{tabular}

MICU, TICU, and SICU have been defined as Intensive Care Units (Medical, Thoracic, and Surgical).

bupropion and (4) varenicline. The 1-800-QUIT-NOW helpline is available in most states and, depending on the state, offers counselling and free medications to those without insurance. Volunteers were trained to conduct the postdischarge follow-up phone calls (Duffy et al. 2010).

\section{Data collection}

The anonymous survey asked the nurses questions pertaining to the Tobacco Tactics training and implementation at 
Table 2 Tobacco Tactics protocol

Tobacco Tactics pharmaceutical management protocol

1. Recommend nicotine replacement (patch, gum or lozenge) if:

Never used patch, gum or lozenge before

Used patch, gum or lozenge successfully in the past

(smoke-free >three months)

2. Recommend bupropion if:

Failed nicotine replacement monotherapy in the past (smoke-

free $<$ three months)

Patch, gum or lozenge intolerant (i.e. rash, etc.)

History of depression or currently has depressive symptoms

3. Recommend combination nicotine replacement (patch, gum or lozenge) and bupropion if:

Failed nicotine replacement and bupropion monotherapy in the past

4. Recommend varenicline if:

Intolerance or treatment failure to nicotine replacement and bupropion

Tobacco Tactics behavioural management protocol

1. Assess whether patient interested in quitting

2. If patient not interested, leave brochure at bedside

3. If patient interested, leave brochure and arrange for patient to view videotape

4. After videotape, provide patient with patient manual to read if able

5. Using patient manual, assist patient with behavioural

intervention including:

Self assessment

Smoker type

Smoking costs

Handling cravings

Relapse prevention

Medication options

6. Along with patient, identify and arrange for cessation medications (see pharmaceutical protocol)

7. Arrange for follow-up calls (Duffy et al. 2010)

the VA including: (1) satisfaction with the material presented (rated on a five-point Likert scale ranging from extremely satisfied to extremely dissatisfied), (2) perception of understanding the elements of the smoking cessation intervention (rated on a five-point Likert scale ranging from strongly agree to strongly disagree), (3) confidence in abilities to provide tobacco cessation services (rated on a fivepoint Likert scale ranging from extremely confident to not at all confident), (4) perceived level of importance of providing services (rated on a five-point Likert scale ranging from very important to not at all important), (5) anticipated barriers to implementation (yes/no) and (6) 'Is there anything that would make it easier for you to implement the smoking cessation intervention in your unit?' (open ended). Participants were also asked questions about their own smoking status (current, former and never smoker) and demographic information (age, gender, race and educational level) and type of work unit.
Information on provision of services pretraining and posttraining was obtained from the 15 -month post-training survey that asked respondents about their pretraining behaviour retrospectively and their current (post-training) behaviour with the following two questions: (1) 'Do you personally provide smoking cessation services to veterans?' and (2) 'Prior to the Tobacco Tactics training, did you personally provide smoking cessation services to veterans?' This information was used to compare preintervention to postintervention selfreported cessation delivery rates.

\section{Data analysis}

Frequencies were examined for all variables. To examine whether or not perceived self-confidence in and importance of delivering the intervention decreased with increased time post-training, the Kruskal-Wallis test was used to compare the proportions of responses to each question from the twomonth post-training surveys vs. the 15 -month post-training surveys. The Kruskal-Wallis test, a nonparametric test for non-normally distributed data (Corder \& Foreman 2009), was used to compare responses from two different samples. This test was applied to check whether the two- and 15month post-training survey proportions were different. To examine whether or not provision of services changed in response to the training, chi-square tests were used to compare the proportion of nurses' reporting that they provided smoking cessation services prior to receiving the Tobacco Tactics training compared to after they received the Tobacco Tactics training. Moreover, chi-square tests were used to compare the proportion of nurses' reporting that they provided smoking cessation services in a cross-sectional pretraining study in 2004 to 15 months post-training in 2007.

As all of the respondents did not answer all of the questions, the sample size varied for different results. Those with missing data for a particular question were not included in the analysis. Values for $p<0.05$ are reported. Two members of the research team categorised the comments by topic. Data analysis for the purposes of this study was conducted using (SAS Version 9.2; SAS Institute Inc., Cary, NC, USA).

\section{Results}

At 15 months post-training, the vast majority (over 85\%) of staff felt at least moderately, very or extremely confident in providing smoking cessation services and felt that providing these services was important or very important. Likewise, the vast majority (nearly 90\%) were somewhat or extremely satisfied with the training and agreed or strongly 
agreed that they had a good understanding of the elements of the intervention.

Nearly three-quarters of the participants indicated that they anticipated barriers to implementing the Tobacco Tactics intervention. The most commonly cited barriers included patients not being interested and lack of time. Common suggestions for improvement, as indicated with the open-ended question, included designating key personnel to perform or coordinate smoking cessation interventions $(n=10)$, having resources readily available $(n=10)$, planning scheduled sessions for counselling $(n=7)$ and improving the documentation template to improve usability $(n=4)$.

Satisfaction with the training and having a good understanding of the intervention were positively associated with perceived confidence in providing cessation services and feeling that delivering cessation interventions was important. Those who were extremely satisfied with the training were significantly more confident in their abilities to provide smoking cessation interventions $(p<0.0001)$ and to think it was important to provide such services on their units ( $p=0.0002)$ (Table 3). Moreover, those who strongly agreed to have a good understanding of the elements of the smoking cessation intervention were also significantly more confident in their abilities to provide smoking cessation interventions $(p<0.0001)$ and to think it was important to provide such services on their units $(p=0.0083$; Table 3$)$. There were no significant differences in whether or not participants anticipated barriers to implementing the intervention, smoking status, age, sex, race, or education level or type of work unit and perceived self-confidence in and importance of delivering the intervention. The number of participants in this study who currently smoked was very small $(n=8,4.8 \%$ participants who completed 15 -month post-training surveys), and there was no statistically significant difference between smoking status and self-report of pre or post-training provision of smoking cessation services. In fact, the reported rates of smoking cessation service provision were higher among the current smokers $(75 \%, n=3$, before training, $100 \%, n=4$, after training) as compared to former smokers $(56.7 \%, n=17$ and $77.4 \%, n=24$, respectively) or never smokers $(57.1 \%, n=28$ and $89.6 \%, n=43$, respectively) (Table 4). Additionally, there were no significant differences in perceived self-confidence in or level of importance of delivering the intervention when comparing responses from two-month vs. 15-month post-training surveys.

Significant differences in self-reported delivery of cessation services pre and postintervention are shown in Table 5.

Table 4 Association between smoking status and self-reported delivery of cessation services

\begin{tabular}{lcc}
\hline & $\begin{array}{l}\text { Prior to training, } \\
\text { provided smoking } \\
\text { cessation to } \\
\text { veterans, } n(\%)\end{array}$ & $\begin{array}{l}\text { Since receiving the } \\
\text { training, now provide } \\
\text { smoking cessation to } \\
\text { veterans, } n(\%)\end{array}$ \\
\hline $\begin{array}{l}\text { Smoking status } \\
\text { Current }\end{array}$ & $3(75.0)$ & $4(100 \cdot 0)$ \\
$\begin{array}{l}\text { Former } \\
\text { Never }\end{array}$ & $17(56.7)$ & $24(77.4)$ \\
$p$-value & $28(57.1)$ & $43(89.6)$ \\
\hline
\end{tabular}

Based on Kruskal-Wallis test (Corder \& Foreman 2009).

Table 3 Associations between nurses satisfaction with and having understanding of the Tobacco Tactics training and perceived confidence in and importance of delivering cessation services

\begin{tabular}{|c|c|c|c|c|c|c|}
\hline & \multicolumn{3}{|c|}{$\begin{array}{l}\text { How confident are you in your abilities to } \\
\text { provide smoking cessation services to } \\
\text { smokers? }\end{array}$} & \multicolumn{3}{|c|}{$\begin{array}{l}\text { How important do you think it is to } \\
\text { provide the smoking cessation } \\
\text { intervention in your unit? }\end{array}$} \\
\hline & Extremely & Very & $\begin{array}{l}\text { Moderately/ } \\
\text { somewhat/ } \\
\text { not at all }\end{array}$ & Extremely & Very & $\begin{array}{l}\text { Neutral/ } \\
\text { not very/ } \\
\text { not at all }\end{array}$ \\
\hline \multicolumn{7}{|c|}{ In general, how satisfied were you with the material presented? (\%) } \\
\hline Extremely satisfied & $20(24.4)$ & $45(54.9)$ & $17(20 \cdot 7)$ & $50(61 \cdot 0)$ & $25(30 \cdot 5)$ & $7(8 \cdot 5)$ \\
\hline $\begin{array}{l}\text { Somewhat satisfied/neutral/undecided/ } \\
\text { somewhat not/extremely not satisfied }\end{array}$ & $3(4 \cdot 8)$ & $19(30 \cdot 7)$ & $40(64 \cdot 5)$ & $16(26 \cdot 2)$ & $32(52 \cdot 5)$ & $13(21 \cdot 3)$ \\
\hline Chi-square statistic (DF) & & $30 \cdot 21(2)$ & & & $17 \cdot 5(2)$ & \\
\hline$p$-value & & $<0 \cdot 0001$ & & & 0.0002 & \\
\hline \multicolumn{7}{|c|}{ Do you feel you have a good understanding of the elements of the smoking cessation intervention? (\%) } \\
\hline Strongly agree & $19(46 \cdot 3)$ & $18(43 \cdot 9)$ & $4(9 \cdot 8)$ & $27(65 \cdot 9)$ & $9(22 \cdot 0)$ & $5(12 \cdot 2)$ \\
\hline Agree/neutral/disagree/strongly disagree & $4(3 \cdot 9)$ & $46(44 \cdot 7)$ & $53(51 \cdot 5)$ & $39(38 \cdot 2)$ & $48(47 \cdot 1)$ & $15(14 \cdot 7)$ \\
\hline Chi-square statistic (DF) & & $46 \cdot 0(2)$ & & & $9.6(2)$ & \\
\hline$p$-value & & $<0 \cdot 0001$ & & & $0 \cdot 0083$ & \\
\hline
\end{tabular}

Bold values are significant at $p<0.01$. 
Table 5 Self-reported delivery of cessation services pre and postintervention

\begin{tabular}{|c|c|c|c|}
\hline & $\begin{array}{l}\text { Preintervention } \\
\text { self-reported } \\
\text { cessation } \\
\text { delivery } \\
\text { rates, } n(\%)\end{array}$ & $\begin{array}{l}\text { Postintervention } \\
\text { self-reported } \\
\text { cessation } \\
\text { delivery } \\
\text { rates, } n(\%)\end{array}$ & $p$-value \\
\hline $\begin{array}{l}\text { Among nurses } \\
\text { who participated } \\
\text { in the Tobacco } \\
\text { Tactics } \\
\text { intervention }\end{array}$ & $40(57 \cdot 1)$ & $60(85 \cdot 7)$ & 0.0002 \\
\hline $\begin{array}{l}\text { Comparison } \\
\text { between a cross- } \\
\text { section of nurses } \\
\text { surveyed in } 2004 \\
\text { (preintervention) } \\
\text { and the Tobacco } \\
\text { Tactics nurses } \\
\text { postintervention }\end{array}$ & $30(43.5)$ & $60(85 \cdot 7)$ & $<0.0001$ \\
\hline
\end{tabular}

Among the nurses surveyed 15 months post-training, 57.1\% $(n=40)$ reported delivering cessation interventions prior to the Tobacco Tactics training compared with $85.7 \%(n=60)$ at the 15 -month post-training follow-up $(p=0 \cdot 0002)$. The $85.7 \%$ self-reported postintervention delivery of cessation services was also significantly higher than the published 2004 pretraining study (Duffy et al. 2008) where only $43.5 \% \quad(n=30)$ indicated that they personally provided smoking cessation services to veterans $(p<0 \cdot 0001)$.

\section{Discussion}

With as little as a one-hour training session, the proportion of nurses self-reporting the provision of cessation services significantly increased from preintervention to postintervention, suggesting that the Tobacco Tactics training increased nurses' likelihood of providing smoking cessation services. Implementing standard protocols, which have been shown to be effective in increasing smoking cessation counselling interventions provided by nurses (Nolan et al. 2005), likely enhanced service delivery. When smokers are offered cessation services, particularly in inpatient settings, a significant number of smokers quit (Rigotti et al. 2002). As knowledge and satisfaction are associated with confidence and importance, careful consideration of smoking cessation training and protocol development is necessary.

Post-training, most nurses were confident providing cessation services compared to $60 \%$ in the prior preintervention study (Duffy et al. 2008). Moreover, post-training, there were no significant changes in perceived confidence in and perceived importance of delivering the intervention between the two-month surveys and the 15-month surveys, suggesting sustainability over time. Those who were more satisfied with and had a better understanding of the Tobacco Tactics intervention had significantly higher perceived confidence scores and importance scores related to providing the intervention. Providers who feel more confident in their ability to do what is expected (self-efficacy), recognise the need and importance for the intervention and have the requisite skills are more likely to implement a programme at higher levels of fidelity (Durlak \& DuPre 2008). Increased selfconfidence and perceived importance of delivering cessation interventions have been shown to be associated with a greater likelihood that cessation interventions will be delivered (Laschinger \& Tresolini 1999, Borrelli et al. 2001).

While three-quarters of the respondents anticipated barriers to implementation, anticipated barriers did not affect ones' perceived confidence in or importance of delivering the interventions. There was the perception among over half of the nurses that patients were not interested in cessation services. Yet, data have shown that over $80 \%$ of smokers and $70 \%$ of Veteran smokers (Duffy et al. 2008) want to quit (Cameron et al. 1994). Patients have reported social support as an important barrier to successful smoking cessation (Macnee \& Talsma 1995a), and nursing assessment of smoking patients' perceived barriers to smoking cessation and self-efficacy can support smoking cessation (Macnee \& Talsma 1995b). Similar to other studies (McCarty et al. 2001, Sarna et al. 2001), lack of time remains a barrier, and several nurses suggested there be a dedicated tobacco cessation counsellor. Yet, designated cessation counsellors are expensive and may have difficulty locating patients in busy inpatient settings, whereas nurses have ready access to patients, have the most one-on-one contact with patients and are positioned to coordinate smoking cessation interventions around other patient care needs.

Contrary to the literature (Braun et al. 2004), no significant differences were seen between current, former and never smokers related to perceived confidence in and importance of delivering tobacco cessation interventions, perhaps because $<10 \%$ of the nurses in this study were current smokers. Age, sex, race and educational level (shown to be associated with nurses smoking and views about smoking) (Duffy et al. 2008, Essenmacher et al. 2009) or type of work unit was not associated with perceived confidence in and importance of delivering cessation services. These data suggest that nurses of all ages, sexes, races and educational levels on any work unit can acquire this skill. 


\section{Limitations}

The survey was conducted with all inpatient nurses in a particular facility and is thereby only generalisable to those staff. The response rate was low $(45 \%)$, albeit not particularly low compared to other studies surveying nurses on tobacco cessation programmes in clinical practice (Sarna et al. 2001). Despite these limitations, this study is one of only a few that provide valuable process evaluation about the implementation of nurse-delivered tobacco cessation interventions that can be used to identify potential and actual influences on the progress and effectiveness of implementation efforts (Stetler et al. 2006).

\section{Conclusions}

Nurses who participated in the Tobacco Tactics intervention reported high perceived confidence in and importance of delivery of cessation interventions. Self-reported delivery of cessation services increased after the implementation of the Tobacco Tactics educational programme, which is likely to enhance quit rates.

\section{Relevance to clinical practice}

The nurse-delivered Tobacco Tactics intervention meets the standards set by the Joint Commission released in July 2011 including provision of treatment during the hospital stay, provision of treatment at discharge and one-month postdischarge follow-up. This study showed that training nurses in delivering the Tobacco Tactics intervention increased delivery of services. Nurse-delivered cessation interventions, such as the Tobacco Tactics intervention, have the potential to increase quit rates and decrease morbidity and mortality among patient populations. The Tobacco Tactics intervention is currently being tested in a large National Institutes of Health-funded study outside the VA system.

\section{Acknowledgements}

This study was funded by the Department of Veterans Affairs (SDP 06-003).

\section{Disclosure}

The authors have confirmed that all authors meet the ICMJE criteria for authorship credit (www.icmje.org/ ethical_1author.html), as follows: (1) substantial contributions to conception and design of, or acquisition of data or analysis and interpretation of data; (2) drafting the article or revising it critically for important intellectual content; and (3) final approval of the version to be published.

\section{Conflict of interest}

No conflict of interest has been declared by the authors.

\section{References}

Borrelli B, Hecht JP, Papandonatos GD, Emmons KM, Tatewosian LR \& Abrams DB (2001) Smoking-cessation counseling in the home. Attitudes, beliefs, and behaviors of home healthcare nurses. American Journal of Preventive Medicine 21, 272277.

Braun BL, Fowles JB, Solberg LI, Kind EA, Lando H \& Pine D (2004) Smokingrelated attitudes and clinical practices of medical personnel in Minnesota. American Journal of Preventive Medicine 27, 316-322.

Cameron R, Zanna MP \& Walker R (1994) Creating a Community Profile: An Approach to Planning and Supporting Intervention Activities. Health and Canadian Society.

Campbell S, Pieters K, Mullen KA, Reece R \& Reid RD (2011) Examining sustainability in a hospital setting: case of smoking cessation. Implementation Science 6, 108.

Casey D (2007) Using action research to change health-promoting practice. Nursing \& Health Sciences 9, 5-13.

Centers for Disease Control and Prevention (2007) Cigarette smoking among adults-United States, 2006. MMWR Morbidity and Mortality Weekly Report 56, 1157-1161.

Corder GW \& Foreman DI (2009) NonParametric Statistics for Non-Statisticians: A Step-by-Step Approach. John Wiley \& Sons, Hoboken, NJ.

Department of Veterans Affairs Veterans Health Administration (2006) Office of 585 the Assistant Deputy Under Secretary for Health for Policy and Planning. 2005 Survey of Veteran Enrollees' Health and Reliance Upon
VA. Department of Veterans Affairs, Veterans Health Administration, Washington, DC.

Duffy SA, Reeves P, Hermann C, Karvonen C \& Smith P (2008) In-hospital smoking cessation programs: what do VA patients and staff want and need? Applied Nursing Research 21, 199 206.

Duffy SA, Karvonen-Gutierrez CA, Ewing LA, Smith PM \& Veterans Integrated Services Network (VISN) 11 Tobacco Tactics Team (2010) Implementation of the Tobacco Tactics Program in the Department of Veterans Affairs. Journal of General Internal Medicine 25(Suppl. 1), 3-10.

Durlak JA \& DuPre EP (2008) Implementation matters: a review of research on the influence of implementation on program outcomes and the factors 
affecting implementation. American Journal of Community Psychology 41, 327-350.

Essenmacher C, Duffy SA, KarvonenGutierrez C \& Lynch-Sauer J (2009) Staff attitudes toward the delivery of tobacco cessation services in a primarily psychiatric Veterans Affairs hospital. Archives of Psychiatric Nursing 23, 231-242.

Joint Commission (2011) Tobacco Treatment Measures (TTM). Vol. 2012. Available at: http://www.jointcommiss ion.org/assets/1/6/Tobacco\%20Treatm ent $\% 20$ Measures\%20List1.PDF (accessed 14 March 2012).

Laschinger HK \& Tresolini CP (1999) An exploratory study of nursing and medical students health promotion counselling self-efficacy. Nurse Education Today 19, 408-418.

Macnee CL \& Talsma A (1995a) Development and testing of the barriers to cessation scale. Nursing Research 44, 214-219.

Macnee CL \& Talsma A (1995b) Predictors of progress in smoking cessation. Public Health Nursing 12, 242-248.

McCarty MC, Hennrikus DJ, Lando HA \& Vessey JT (2001) Nurses' attitudes concerning the delivery of brief cessation advice to hospitalized smokers. Preventive Medicine 33, 674-681.

Nolan E, VanRiper S, Talsma A, Mageno L, Richter A, Kearly G, Kendrick C, Leggett S, Crissey J, Tsai T, Blackford G, Shlafer J, Montoye C, Mehta R, Koelling T, Cody R \& Eagle K (2005) Rapid-cycle improvement in quality of care for patients hospitalized with acute myocardial infarction or heart failure: moving from a culture of missed opportunity to a system of accountability. Journal of Cardiovascular Management 16, 14-19.

Office of Quality and Performance (2001) Health Behaviors of Veterans in the VHA: Tobacco Use. 1999 Large Health Survey of Enrollees. Veterans Health Administration, Washington, DC.

Rice VH \& Stead LF (2008) Nursing interventions for smoking cessation. Cochrane Database Systematic Reviews (1), CD001188.

Rigotti NA, Munafo MR, Murphy MFG \& Stead LF (2002) Interventions for smoking cessation in hospitalised patients. Cochrane Database Systematic Reviews (4), CD001837.
Sarna L, Wewers ME, Brown JK, Lillington L \& Brecht ML (2001) Barriers to tobacco cessation in clinical practice: report from a National Survey of Oncology Nurses. Nursing Outlook 49, 166-172.

Smith PM, Kraemer HC, Miller NH, DeBusk RF \& Taylor CB (1999) Inhospital smoking cessation programs: who responds, who doesn't? Journal of Consulting and Clinical Psychology 67, 19-27.

Stetler CB, Legro MW, Wallace CM, Bowman C, Guihan M, Hagedorn H, Kimmel B, Sharp ND \& Smith JL (2006) The role of formative evaluation in implementation research and the QUERI experience. Journal of General Internal Medicine 21(Suppl. 2), S1-S8.

Taylor CB \& Curry SJ (2004) Implementation of evidence-based tobacco use cessation guidelines in managed care organizations. Annals of Behavioral Medicine 27, 13-21.

Wewers ME, Kidd K, Armbruster D \& Sarna L (2004) Tobacco dependence curricula in U.S. baccalaureate and graduate nursing education. Nursing Outlook 52, 95-101.

The Journal of Clinical Nursing (JCN) is an international, peer reviewed journal that aims to promote a high standard of clinically related scholarship which supports the practice and discipline of nursing.

For further information and full author guidelines, please visit JCN on the Wiley Online Library website: http:// wileyonlinelibrary.com/journal/jocn

\section{Reasons to submit your paper to JCN:}

High-impact forum: one of the world's most cited nursing journals, with an impact factor of 1.316 - ranked 21/101 (Nursing (Social Science)) and 25/103 Nursing (Science) in the 2012 Journal Citation Reports ${ }^{\circledR}$ (Thomson Reuters, 2012).

One of the most read nursing journals in the world: over 1.9 million full text accesses in 2011 and accessible in over 8000 libraries worldwide (including over 3500 in developing countries with free or low cost access).

Early View: fully citable online publication ahead of inclusion in an issue.

Fast and easy online submission: online submission at http://mc.manuscriptcentral.com/jcnur.

Positive publishing experience: rapid double-blind peer review with constructive feedback.

Online Open: the option to make your article freely and openly accessible to non-subscribers upon publication in Wiley Online Library, as well as the option to deposit the article in your preferred archive. 